\title{
Delayed degradation according to the location of fixation with using an absorbable plate
}

Tae Ho Kim,

Seok Joo Kang,

Hook Sun

Department of Plastic and Reconstructive Surgery, Busan Paik Hospital, Inje University School of Medicine, Busan, Korea

\begin{abstract}
Background: The ideal absorbable plating system should provide sufficient rigidity and then be absorbed within a timely manner. The Resorb- $X$ has been recently developed as a plating system with a mixture ratio of 50:50 poly(D, L-lactide).

Methods: We present seven of 121 patients who experienced delayed degradation with this absorbable plate. One hundred twenty-one patients with facial bone fracture underwent surgical treatment from March 2011 to March 2015, and rigid fixation was achieved with the Resorb-X.

Results: Of 121 patients, seven (5.8\%) developed complications at the surgical sites. Six of 102 cases underwent fixation of the infraorbital rim and one of 73 underwent fixation of the frontozygomatic buttress; the other sites of fixation did not develop delayed degradation. Foreign body granuloma developed at the earliest by postoperative 20 months and at the latest by postoperative 28 months (average, 23.5 months).

Conclusion: We observed that the use of absorbable plates in incision sites or areas with thin skin can increase the possibility of delayed degradation. When performing surgery in these areas, the normal skin above the fixed location should be covered sufficiently.
\end{abstract}

Keywords: Facial bones / Orbital implants / Bone plates

\section{INTRODUCTION}

Absorbable materials are widely used for the surgical treatment of facial fractures. Of these, the Resorb-X (KLS Martin, Tuttlingen, Germany) has been recently developed as a plating system with a mixture ratio of 50:50 poly(D, L-lactide) (PDLLA). In vivo light microscopic examination demonstrates that pure PDLLA disappears from the extracellular space within 72 weeks of implantation. Moreover, PDLLA maintains $90 \%$ of its bending strength at 6 weeks after implantation and $60 \%$ of it at 12 weeks [1]. The ideal plating system should provide sufficient rigidity, biocompatibility, and stability to allow bone healing, and then be absorbed within a timely manner. If the absorbable plates fail to degrade completely

\section{Correspondence: Seok Joo Kang}

Department of Plastic and Reconstructive Surgery, Busan Paik Hospital, Inje University School of Medicine, 75 Bokji-ro, Busanjin-gu, Busan 47392, Korea

E-mail: sonydr@naver.com

Received June 8, 2018 / Revised June 10, 2018 / Accepted June 11, 2018 or undergo polymeric breakdown beyond the maximum metabolic clearance rate, the remnant material can become a nidus for an inflammatory foreign body reaction. Herein, we present seven of 121 patients who experienced delayed degradation with the absorbable Resorb-X.

\section{METHODS}

This study was reviewed and approved by the Ethics Review Board of the Inje University Health Center. One hundred twentyone outpatients with facial fractures were treated with the Resorb$\mathrm{X}$ by two plastic surgeons (SJK and HS) from March 2011 to March 2015. The subjects were 96 men and 25 women, and their ages ranged from 11 to 79 years (average age, 37.8 years). We also classified the frequency of delayed degradation based on the location of fixation (Table 1 ).

Of 121 patients, seven (5.8\%) developed complications at the 
surgical sites (Table 2). All patients visited the hospital with chief complaints of weakness, pain, swelling, and palpable mass at the surgical site. Initially, conservative treatments, such as an intralesional steroid injection and oral steroid administration, were performed for about 1 month, and the patients were followed up for observation. However, their symptoms did not improve, and they continued to feel discomfort. Therefore, mass excision of the affected area and histopathologic examination was performed.

\section{RESULTS}

Eighty-one patients (66.9\%) had zygomaticomaxillary complex (ZMC) fracture, 19 (15.7\%) had maxillary fracture, 13 (10.7\%) had infraorbital rim fracture, and eight (6.6\%) had Lefort II fracture.

Table 1. Patient demographic characteristics

\begin{tabular}{lc} 
Characteristics & Value \\
Total & 121 \\
Sex, no. (\%) & \\
$\quad$ Male & $96(79)$ \\
Female & $25(17)$ \\
\hline Age (yr), mean (range) & $37.8(11-79)$ \\
Diagnosis (FBG/total) & \\
ZMC fracture & $4 / 81$ \\
Maxillary fracture & $0 / 19$ \\
Infraorbital rim fracture & $2 / 13$ \\
LeFort II fracture & $1 / 8$ \\
Site of FBG/fixation site of the Resorb-X & \\
Zygomaticomaxillary buttress & $0 / 95$ \\
Frontozygomatic buttress & $1 / 73$ \\
Infraorbital rim & $6 / 102$ \\
Nasomaxillary complex & $0 / 91$ \\
\hline
\end{tabular}

FBG, foreign body granuloma; ZMC, zygomaticomaxillary complex.
The sites of fixation were as follows: the zygomaticomaxillary buttresses, 95; infraorbital rims, 102; nasomaxillary complex, 91; and frontozygomatic buttresses, 73 (Table 1).

Of 121 patients, seven (5.8\%) developed complications at the surgical sites; of those, five were men and two were women (average age, 27.1 years) (Table 2). Four of seven patients with complications underwent surgery for ZMC fracture, two for infraorbital rim fracture, and one patient for Lefort II fracture. Six of 102 cases underwent fixation of the infraorbital rim and one of 73 underwent fixation of the frontozygomatic buttress; the other sites of fixation did not develop delayed degradation. Foreign body granuloma (FBG) developed at the earliest by postoperative 20 months and at the latest by postoperative 28 months (average, 23.5 months).

\section{Case 1}

The first case was a 29-year-old man who received the Resorb-X because of ZMC fracture 27 months ago. He visited the hospital because of a mass under his eye and mild pain for 1 month (Fig. 1). The conservative treatments were ineffective, so he underwent surgical management. We made a subciliary incision and removed the pale yellow mass in the infraorbital rim (Fig. 2). On histopathologic examinations, there were eosinophilic, amorphous foreign body materials (Fig. 3A). There was also chronic, granulomatous inflammation with several multinucleated giant cells containing transparent foreign materials (Fig. 3B). On the 3rd day postoperatively, he reported that the palpable mass, pain, and swelling had disappeared. There was also no discomfort or feeling of irritation after 6-month follow-up. (Fig. 4).

Table 2. Characteristics of the seven patients with delayed degradation

\begin{tabular}{cllcll} 
Patient no. & Sex/age $(\mathrm{yr})$ & \multicolumn{1}{c}{ Diagnosis } & Onset $(\mathrm{mo})$ & Site of fixation & Treatment \\
\hline 1 & Male/36 & ZMC Fx & 24 & Frontozygomatic buttress & Operation \\
2 & Male/29 & ZMC Fx & 28 & Infraorbital rim & Operation \\
3 & Male/28 & ZMC Fx & 27 & Infraorbital rim & Operation \\
4 & Male/31 & ZMC Fx & 21 & Infraorbital rim & Operation \\
5 & Male/20 & Infraorbital rim Fx & 20 & Infraorbital rim & Operation \\
6 & Female/24 & Infraorbital rim Fx & 22 & Infraorbital rim & Operation \\
7 & Female/22 & LeFort II Fx & 23 & Infraorbital rim & Operation \\
\hline
\end{tabular}

ZMC, zygomaticomaxillary complex; Fx, fracture. 


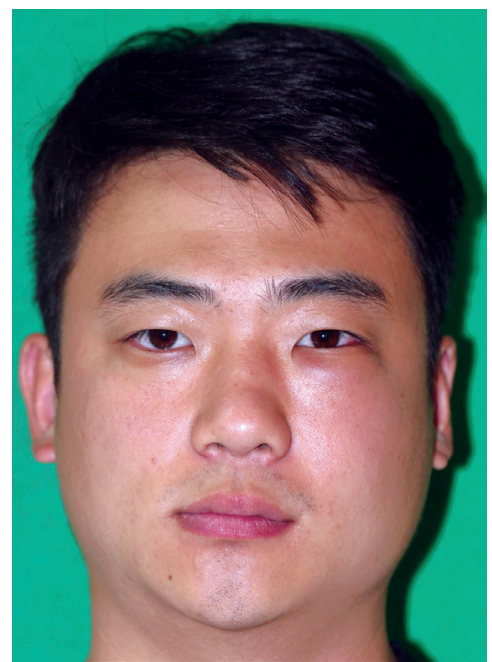

Fig. 1. Preoperative photograph. A 29-year-old man with hardness and swelling in the infraorbital region.

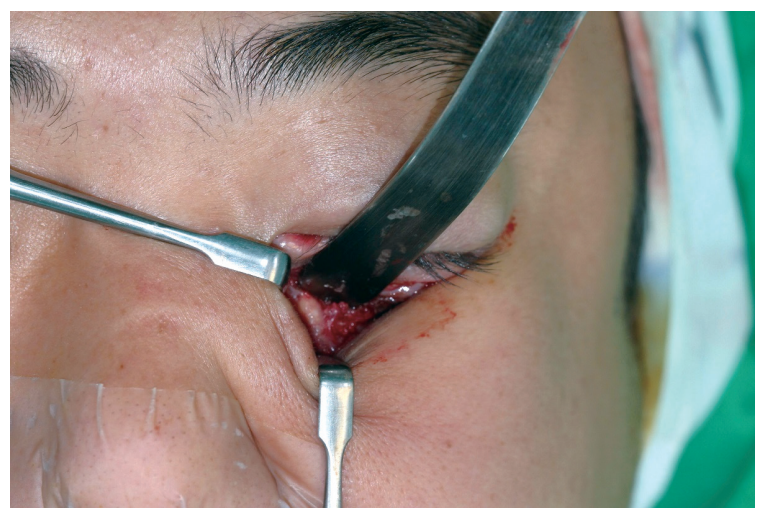

Fig. 2. Intraoperative photograph. The plates are replaced with encapsulated, spongy materials containing yellow, grainy, cloudy liquid.

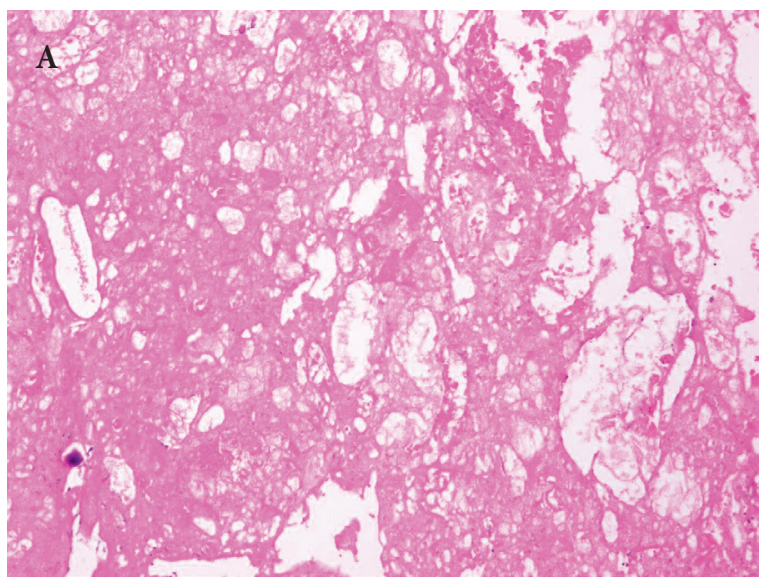

\section{Case 2}

A 24-year-old man underwent surgical correction of the inferior orbital rim fracture using Resorb X and screws, for which we made a subciliary incision and an intraoral one. At a 21-month follow-up, the patient complained of a palpable mass as well as swelling in the left infraorbital area (Fig. 5). Moreover, the patient had no abnormalities of the facial bones, except for soft tissue edema, on computed tomography (CT) scans (Fig. 6). We determined to perform revision surgery. Intraoperatively, we found that the plates were replaced by encapsulated spongy materials containing yellow, grainy and cloudy liquid. After 6-month follow-up, patient had no palpable mass or feeling of irritation in the left infraorbital area (Fig. 7).

\section{DISCUSSION}

Depending on constituents and manufacturing methods, absorbable plates are equipped with a variety of physical and chemical properties. Thus, they have become available for clinical use. poly-L-lactic acid (PLLA) is one of the constituents of absorbable plates, and it has a hydrophobic property and requires an approximately 5 -year absorption period. But polyglycolic acid (PGA) has a crystalline structure with stronger physical properties than PLLA. It is disadvantageous, however, in that it loses its strength within 6 weeks and decomposes quickly with complete absorp-

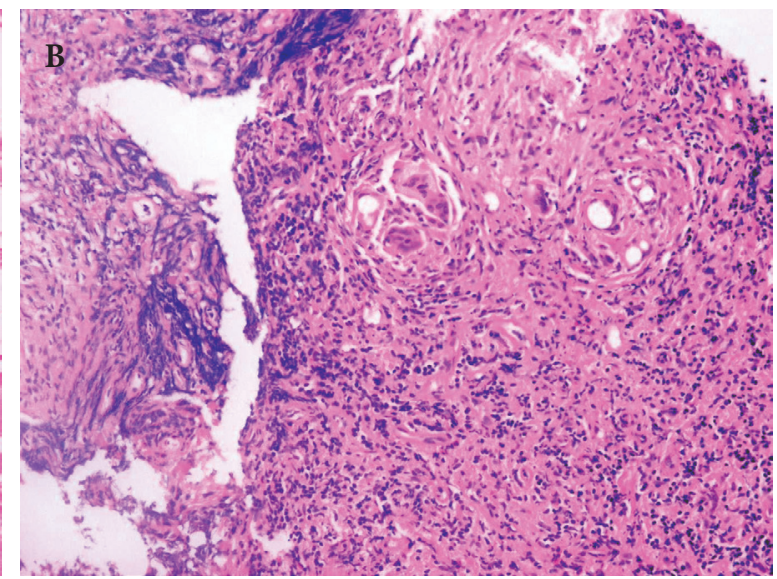

Fig. 3. Histopathologic findings. (A) Eosinophilic, amorphous foreign body materials are shown (H\&E, $\times 100)$. (B) Chronic, granulomatous inflammation with several multinucleated giant cells containing transparent foreign materials is observed $(\mathrm{H} \& \mathrm{E}, \times 200)$. 


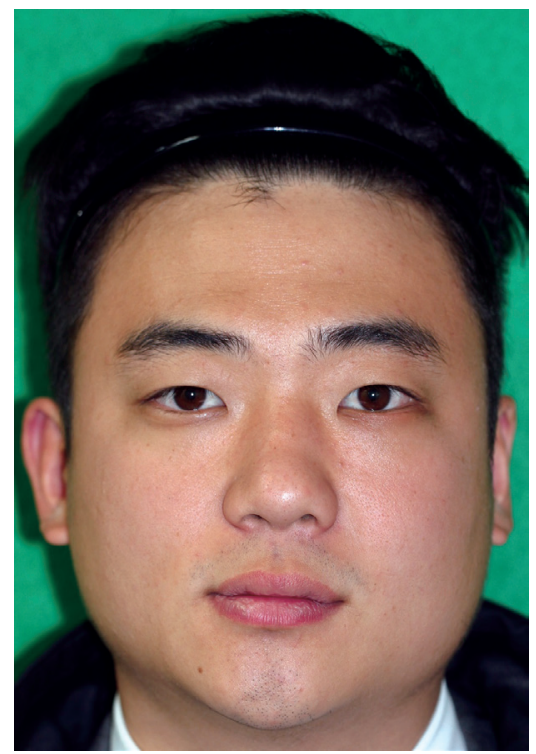

Fig. 4. Postoperative photograph. At the 6-month follow-up, the patient showed great improvement.

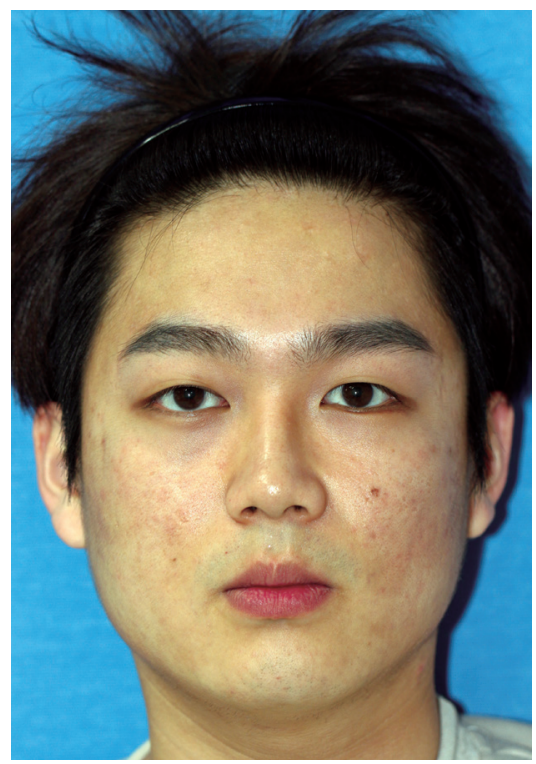

Fig. 5. Preoperative photograph. A 24-year-old man with palpable mass and pain in the infraorbital region.

tion within 3-12 months. Both polymers bind to trimethylene carbonates. Therefore, there is a variability in their flexibility and biocompatibility. The first- and second-generation of absorbable plates have been manufactured using PGA and PLLA, respectively. That is, PLLA has been developed to improve the rapid absorption of PGA. Many studies have reported successful outcomes of
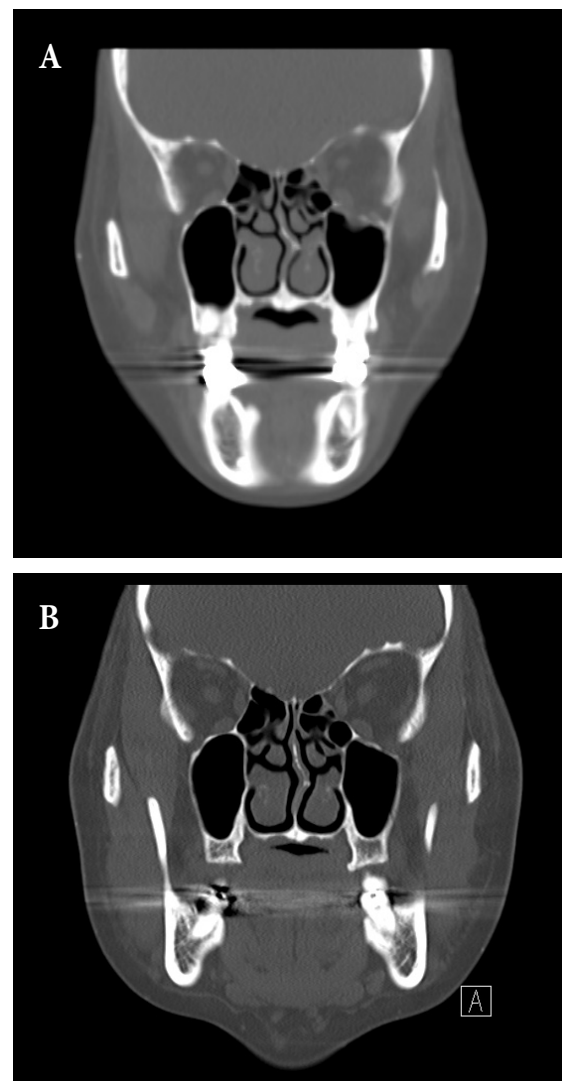

Fig. 6. Pre- and postoperative facial bone computed tomography (CT). (A) Facial bone CT shows left inferior orbital wall fracture. (B) No abnormalities of the facial bones, except for soft tissue edema on facial bone CT.

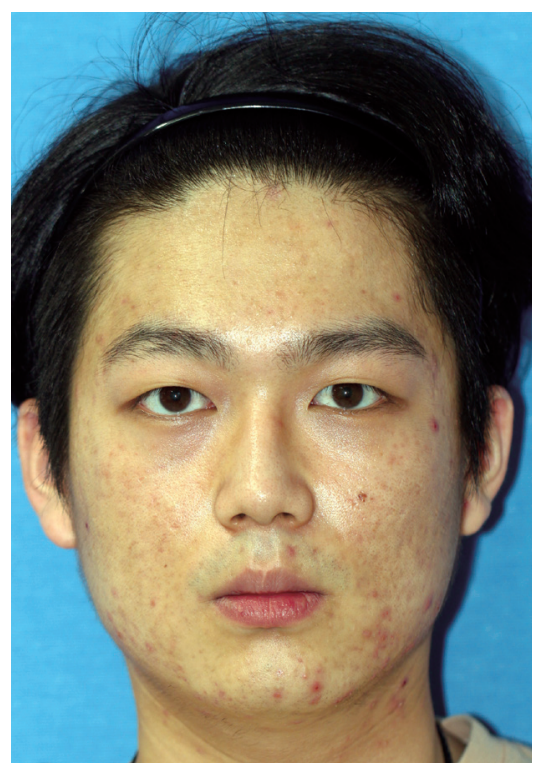

Fig. 7. Postoperative photograph. At the 6-month follow-up, the patient showed great improvement. 
craniofacial surgeries using PLLA until the late 1980s. Thereafter, however, PLLA has been reported to produce such adverse effects as insufficient visibility and palpability, osteolysis and sterile sinus caused by delayed degradation [1].

Resorb $\mathrm{X}$ is a recent resorbable system that is derived solely from 50:50 poly(D,L-lactide) lactide; PDLLA is a purely amorphous compound that degrades in vitro without releasing crystalline byproducts that might be involved in causing foreign body reactions. In vivo light microscopic examination demonstrates that pure PDLLA disappears from the extracellular space within 72 weeks of implantation. Moreover, PDLLA maintains 90\% of its bending strength at 6 weeks after implantation and $60 \%$ at 12 weeks [2]. Degradation of the fixation material starts after an initial incubation period, during which osteogenesis is initiated. The degradation proceeds from the central to peripheral directions within the material. Meanwhile, it is characterized by the volume growth due to water uptake. The thickness of the material is essentially stable until at approximately 8 months of implantation. Then, it increases up to an average of $276 \%$ of baseline [1,2]. According to in vivo studies using rabbits, Resorb X was completely covered with a thick layer of bone material at 12 months of implantation, accompanied by the reduction and remodeling process. On histopathological examinations, there were no polymers and the lumens for the insertion of plate and screw were filled with bone marrow cells [2].

Reichwein et al. [3] reported that $4 \%$ of their total 75 patients developed soft tissue edema 6-8 months after receiving the Resorb-X. Thus, the absorbable plate can be moved during the period when its size is increased by hydrolization even in the normal decomposition process. Therefore, we initially performed conservative treatments, such as an intralesional steroid injection, as we had initially expected a normal absorption process. However, in our first patient, there was no improvement over time, and he complained of more discomfort. Mackool et al. [4] claimed that surgical removal was necessary because the plate with delayed degradation did not decrease in size even after 2 years, and it caused bone resorption. Eventually, we performed surgical removal; our patient's symptom improved, and his appearance improved aesthetically.
In this study, six patients developed FBG in the infraorbital rim and one patient developed it in the frontozygomatic buttress. FBG was not observed at other sites. We believe that there must be a reason why the skin of these areas is thin and conspicuous. It is assumed that a plate fixed through the mouth incision is rapidly absorbed because of the movement of surrounding muscles, such as during mastication, thereby it does not cause issues, or the plate is placed so deep under the skin, it is not visible. This is consistent with many published studies in this series. That is, Salvino [5] reported two cases of delayed absorption of PLLA plate in coronal incision sites in a patient who was concomitantly treated with a metal plate in the intact frontal orbit. Thus, these authors indicated that skin incision areas were associated with the delayed absorption as compared with healthy tissues with adequate blood supplies. Katsuragi et al. [6] also reported that intracerebral FBGs occurred on the temple, a thin-skinned area, as well as incision sites after using PLLA. Thus, the infraorbital rim and frontozygomatic buttress are often close to or in contact with the sites where the plate is fixed and where the skin incision is performed.

In conclusion, delayed degradation associated with this absorbable plate may occur more frequently at the incision site where the skin is thin and the normal layer of the skin is destroyed, and the patient may feel more discomfort at such sites. When performing surgery in these areas, the normal skin above the fixed location should be covered sufficiently. Additionally, the patient should be informed in advance of the operation that the size of the absorbable plate may increase even during the normal decomposition process, creating a palpable mass. The surgeon should be able to distinguish between the normal decomposition process and delayed degradation based on the patient's time of visit, size of the palpable mass, and degree of the patient's symptoms, and choose either conservative or surgical treatment to produce better outcomes.

\section{CONFLICT OF INTEREST}

No potential conflict of interest relevant to this article was reported. 


\section{PATIENT CONSENT}

The patients provided written informed consent for the publication and the use of their images.

\section{REFERENCES}

1. Wood RJ, Petronio JA, Graupman PC, Shell CD, Gear AJ. New resorbable plate and screw system in pediatric craniofacial surgery. J Craniofac Surg 2012;23:845-9.

2. Heidemann W, Gerlach KL. Imaging of biodegradable osteosynthesis materials by ultrasound. Dentomaxillofac Radiol 2002;31:155-8.

3. Reichwein A, Schicho K, Moser D, Seemann R, Poeschl P, Baumann $A$, et al. Clinical experiences with resorbable ultrasonic-guided, anglestable osteosynthesis in the panfacial region. J Oral Maxillofac Surg 2009;67:1211-7.

4. Mackool R, Yim J, McCarthy JG. Delayed degradation in a resorbable plating system. J Craniofac Surg 2006;17:194-7.

5. Salvino MJ. Avoiding pitfalls in craniofacial reconstruction using absorbable plates. J Craniofac Surg 2010;21:213-4.

6. Katsuragi YT, Gomi A, Sunaga A, Miyazaki K, Kamochi H, Arai F, et al. Intracerebral foreign body granuloma caused by a resorbable plate with passive intraosseous translocation after cranioplasty. J Neurosurg Pediatr 2013;12:622-5. 